\title{
Long-distance soliton transmission through ultralong fiber lasers
}

\author{
M. Alcon-Camas, ${ }^{1, *}$ A. E. El-Taher, ${ }^{1}$ H. Wang, ${ }^{1,2}$ P. Harper, ${ }^{1}$ V. Karalekas, ${ }^{1}$ \\ J. A. Harrison, ${ }^{3}$ and J.-D. Ania-Castañón ${ }^{4}$ \\ ${ }^{1}$ Photonics Research Group, School of Engineering and Applied Science, Aston University, Birmingham B4 7ET, UK \\ ${ }^{2}$ Institute of Physics, Nankai University, Tianjin 300071, China \\ ${ }^{3}$ CIP Technologies, Adastral Park, Martlesham Heath, Ipswich IP5 3RE, UK \\ ${ }^{4}$ Instituto de Óptica Daza de Valdés, CSIC, Madrid 28006, Spain \\ *Corresponding author: alconcam@aston.ac.uk
}

Received June 26, 2009; revised August 14, 2009; accepted September 1, 2009; posted September 16, 2009 (Doc. ID 113282); published October 6, 2009

\begin{abstract}
We present the first experimental demonstration (to our knowledge) of long-distance unperturbed fundamental optical soliton transmission in conventional single-mode optical fiber. The virtual transparency in the fiber required for soliton transmission, over 15 complete periods, was achieved by using an ultralong Raman fiber laser amplification scheme. Optical soliton pulse duration, pulse bandwidth, and peak intensity are shown to remain constant along the transmission length. Frequency-resolved optical gating spectrograms and numerical simulations confirm the observed optical soliton dynamics. () 2009 Optical Society of America

OCIS codes: $140.0140,190.0190$.
\end{abstract}

It is well known that optical solitons exist as theoretical solutions of the classic nonlinear Schrödinger equation [1] traditionally used to describe propagation through an optical fiber waveguide. Although exact optical soliton solutions have many potential applications in optical communications [2], laser sources [3] or all-optical signal processing, and signal regeneration [4], they exist only under nondissipative conditions. Unfortunately, in practical optical transmission systems, the intrinsic fiber loss makes unperturbed soliton transmission over long distances impossible even for low-loss media, as the integrity of the soliton pulse relies on the balance between the power-dependent self-phase modulation (SPM) in the transmission fiber and the constant anomalous chromatic dispersion of the fiber.

To overcome this problem, a number of techniques have been used to obtain approximations to the exact fundamental soliton solution. Average solitons find a balance between nonlinearity and dispersion on average over fiber spans where the loss is periodically compensated by lumped amplifiers [2,5]. Dispersionmanaged soliton experiments use periodically alternating dispersion values within the transmission path to obtain periodically stable pulses [6]. Solitons can also be supported in decreasing-dispersion fiber engineered to match the decrease in nonlinearity due to loss [7]. These approaches do produce solitonlike pulses with interesting properties and potential applications but rely on either custom fiber or the exact replication of a loss-cycle-dispersion unit cell between amplification stages to be successful.

An alternative option is to reduce signal power excursion along the fiber by using distributed Raman amplification. This has been shown to support soliton transmission over long distances in recirculating loop experiments [8]. However, to date such demonstrations have been restricted to the case where the fiber spans are shorter than the soliton period owing to there still being considerable power variation along the span length. The experimental results we present here are the first demonstration (to our knowledge) of transmission of fundamental solitons over multiple soliton periods in conventional optical fiber with negligible amplitude or phase distortion. To achieve this we use an ultralong Raman fiber laser transmission scheme that allows signal propagation over long distances in optical fiber with extremely low power level excursions $[9,10]$.

Our initial transmission span consisted of $22 \mathrm{~km}$ of conventional single-mode large effective area silica fiber with a second-order dispersion parameter, $\beta_{2}$, of $-5.8 \mathrm{ps}^{2} / \mathrm{km}$ and a nonlinear coefficient, $\gamma$, of $1.2 \mathrm{~W}^{-1} \mathrm{~km}^{-1}$ at a $1552 \mathrm{~nm}$ operating wavelength. High-reflectivity (99\%) fiber Bragg gratings with a FWHM bandwidth of $0.6 \mathrm{~nm}$ were used to form the cavity. The system was bidirectionally pumped by using two $1365 \mathrm{~nm}$ Raman laser sources. The gratings were designed to give maximum reflectivity at $1455 \mathrm{~nm}$ (the wavelength of the first Raman Stokes shift of our pumps), hence creating a cavity for radiation at that wavelength. The pump and the pulsed signal at $1552 \mathrm{~nm}$ were coupled into the transmission system through 1365/1550 $\mathrm{nm}$ wavelength division multiplexers (WDMs). Using optical time-domain reflectometry it is possible to monitor power excursion at $1550 \mathrm{~nm}$ and manually adjust pump power to fully compensate for fiber attenuation [10].

The sech profile signal pulses, with FWHM of approximately $4 \mathrm{ps}$ and a soliton period of $\sim 1.4 \mathrm{~km}$, were generated by a mode-locked fiber laser (MLFL) operating at $1552 \mathrm{~nm}$. The $10 \mathrm{GHz}$ output pulse train of the fiber laser was modulated down to a repetition rate of $1.25 \mathrm{GHz}$, so that relatively high peak power could be achieved at low average power levels without introducing nonlinear distortion effects from the erbium-doped fiber amplifier (EDFA). Note that the focus of this experiment was to prove the transmission of individual fundamental soliton pulses, and no 


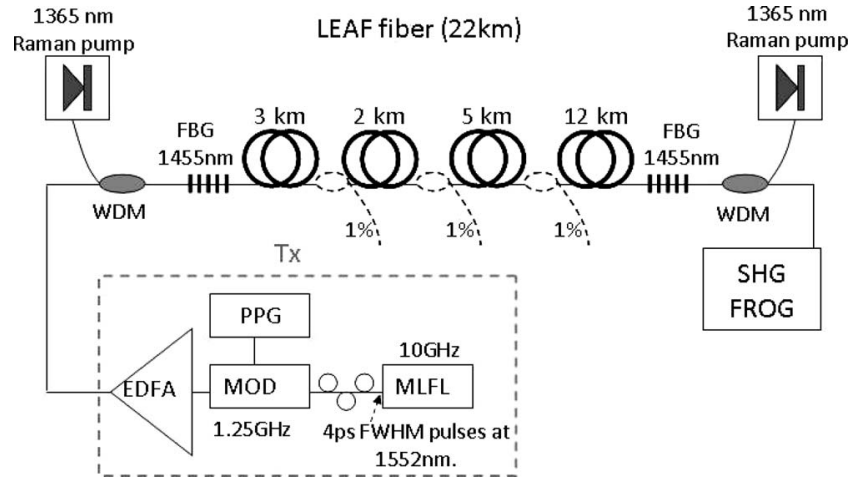

Fig. 1. Experimental setup for observing the soliton transmission at different positions along the fiber.

form of data encoding was applied. The output signal pulses were characterized by using a secondharmonic-generation frequency-resolved optical gating (SHG FROG) [11], which generated complete spectrograms with simultaneous time (horizontal) and wavelength (vertical) information. The average launch power required for fundamental solitons in the ideal case was calculated to be $6.8 \mathrm{dBm}$. However, in practice the required power was slightly higher, $7.8 \mathrm{dBm}$ (pulse energy $4.6 \mathrm{pJ}$ and $1120 \mathrm{~mW}$ of peak power), because of the additional losses within the experimental system (mainly fiber connections). The output of the mode-locked fiber laser was carefully adjusted to obtain a launched pulse intensity profile as close as possible to the typical $\operatorname{sech}^{2}$ of the fundamental soliton.

Similarly, the ultralong Raman fiber laser span was pumped to provide distributed gain as close as possible to ideal transparency. By underpumping or overpumping with respect to this optimal value, it is possible to move from a dispersion-dominated regime to a nonlinearity-dominated regime. In the case of underpumping [Fig. 2(b), curve iii] the pulse displays temporal broadening due to chromatic dispersion. The resulting output pulse [Fig. 2(c), curve iii] will be temporally broadened with respect to the input pulse [Fig. 2(a)]. On the other hand, if the Raman pump power is higher than optimal [Fig. 2(b), i], the pulse experiences a net gain across the span, and SPM dominates, leading to temporal compression. The resulting output pulse [Fig. 2(c), i] is clearly temporally shorter than the launch pulse [Fig. 2(a)]. Optimal pumping [Fig. 2(b), ii] turns the cavity into a virtually nondissipative link with minimum power excursion, in which the balance between the dispersion and the nonlinearity can be maintained. The output [Fig. 2(c), ii] and launched [Fig. 2(a)] pulse spectrograms are similar; therefore its spectral and temporal characteristics are also the same.

The spectrograms, which display the characteristic $\operatorname{sech}^{2}$ shape of a soliton, together with the effectively null intensity variation across the fiber length observed via optical time-domain reflectometry, give a good indication of the dynamics of the fundamental optical soliton, but are not full proof of unperturbed evolution within the fiber span. To experimentally demonstrate that the soliton remains undistorted across the whole length of the fiber, the initial fiber span of $22 \mathrm{~km}(\sim 16$ soliton periods) was divided into four different lengths $(3,2,5$, and $12 \mathrm{~km})$ as schematically depicted in Fig. 1, and spectrograms were taken after each interval. A single 1\% monitoring coupler, with an insertion loss of $0.4 \mathrm{dBm}$, was moved across the range of available positions in the span to measure spectra at different points in the fiber. This set of spectrograms is shown in Fig. 3(a), top row. As expected, the experimental traces closely resemble one another regardless of their position along the fiber. Therefore it can be said that pulse characteristics remain the same for the whole transmission span. Note that the small variations in pulse duration on the experimental trace are caused by the equally small loss variations due to the repositioning of the output coupler.

Otherwise the experimental results are consistent for the whole span, achieving excellent agreement with the numerical simulation results for a fundamental soliton evolution shown on the bottom row of Fig. 3(a). The pulse duration, recovered from the spectrograms, is represented versus distance in Fig. 3(b).

Further experimental work was carried out with span lengths of 50 and $72 \mathrm{~km}$. The experimental

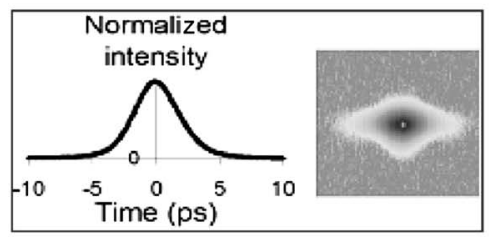

(a)

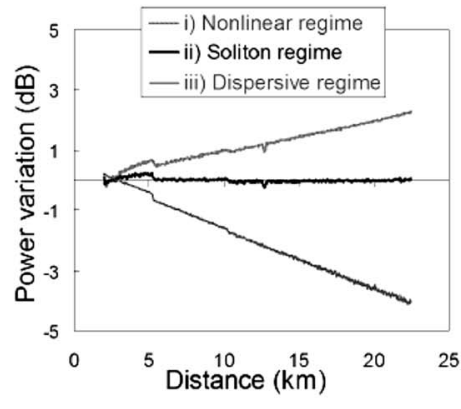

(b)

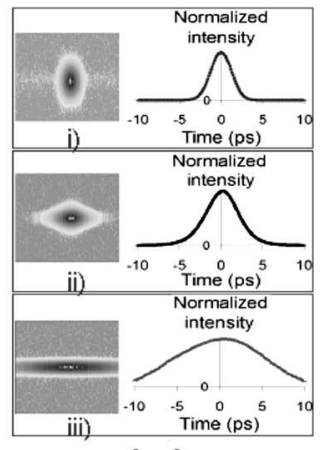

(c)

Fig. 2. (a) Spectrogram and temporal intensity profile of the launched pulse. (b) Power variation profile along a $22 \mathrm{~km}$ fiber span for the (i) nonlinear regime (SPM dominates), $0.83 \mathrm{~W}$ Raman power; (ii) soliton regime (virtually nondissipative), $0.56 \mathrm{~W}$ Raman power; and (iii) dispersive regime (dispersion dominates), 0.29 W Raman power. (c) Spectrogram and temporal intensity profile of the output pulse (i) when SPM dominates, (ii) for the soliton regime, and (iii) when dispersion dominates. 


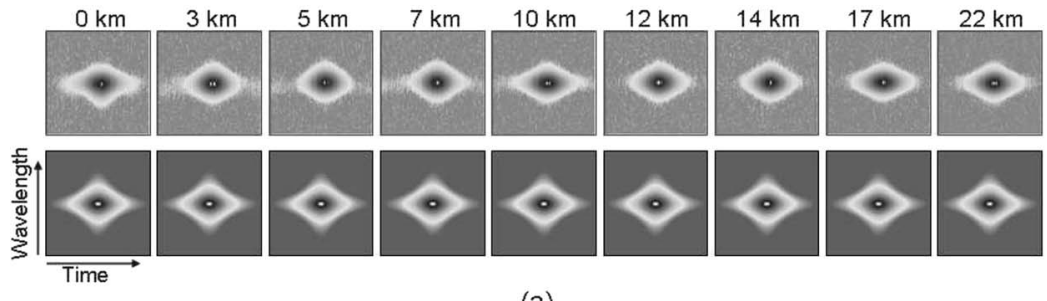

(a)

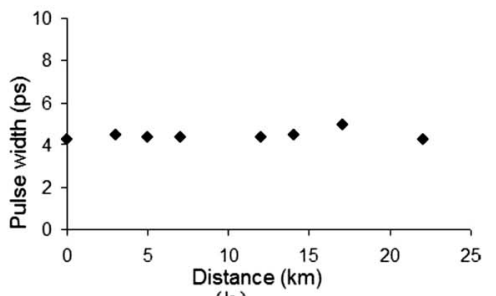

(b)

Fig. 3. (a) Set of experimental (top row) and numerical simulation (bottom row) frequency-resolved optical gating spectrograms at different locations along the fiber span and (b) experimental results for pulse width versus propagation distance.

spectrograms for the launch and output pulses are represented in Fig. 4. Given that a pulse with adequate energy and duration can be expected to evolve naturally into a fundamental soliton while propagating through our virtually nondissipative fiber link, we did not perform an initial optimization of the pulse shape in this case, using instead a 4 ps pseudoGaussian pulse. As expected, the optical pulse evolved toward the typical soliton shape after some initial stabilization. Note that the increasing power excursion of longer fiber spans [10] makes pulse measurements difficult within the fiber span because of the introduction of losses. Therefore, pumping optimization in the transmission span becomes particularly important. In spite of the added difficulties, the spectrograms for the output pulses for the case of $72 \mathrm{~km}$ are still consistent with a soliton profile, indicating that intensity variations within the span are still small enough not to have introduced a significant perturbation to the pulse dynamics.

In conclusion, long-distance transmission of fundamental optical solitons over multiple soliton period without amplitude, temporal, or phase variation has been demonstrated. An ultralong Raman fiber laser amplification scheme was used to provide a virtually nondissipative medium that allowed the signal to propagate at a constant power level as required for soliton stability. Pulse dynamics were monitored and verified by using second-harmonic-generation frequency-resolved optical gating measurements,

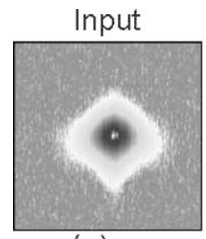

(a)

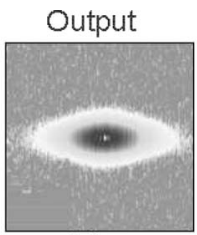

(b)

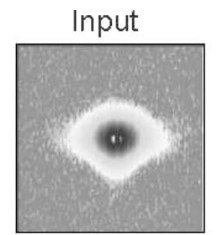

(c)

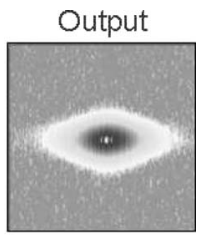

(d)
Fig. 4. Measured spectrograms from launch pulses at (a) $50 \mathrm{~km}$ and (b) $72 \mathrm{~km}$, and output pulses at (c) $50 \mathrm{~km}$ and (d) $72 \mathrm{~km}$. which produce spectrograms that are entirely consistent with those predicted through numerical simulations of fundamental soliton transmission. Our initial experiment shows stability of a 4 ps soliton over a $22 \mathrm{~km}$ span of large effective area fiber (LEAF) a distance equivalent to more than 15 soliton periods. This distance was then extended to more than 50 soliton periods for a $72 \mathrm{~km}$ span. These results, obtained by using conventional fiber, represent an important step toward the possible implementation of practical soliton transmission and optical processing systems.

The authors acknowledge the support of the Engineering and Physical Sciences Research Council (EPSRC) through grant EP/E015646/1, and of the Spanish Ministerio de Ciencia e Innovación (MICINN) through grant TEC2008-05791.

\section{References}

1. V. E. Zakharov and A. B. Shabbat, Sov. Phys. JETP 34, 62 (1972).

2. N. J. Doran and K. J. Blow, IEEE J. Quantum Electron. 19, 1883 (1983).

3. K. Sumimura, T. Ohta, and N. Nishizawa, Opt. Lett. 33 24, 2892 (2008).

4. E. Desurvire, O. Leclerc, and O. Audouin, Opt. Lett. 21 14, 1026 (1996).

5. K. J. Blow and N. J. Doran, IEEE Photon. Technol. Lett. 3, 369 (1991).

6. N. J. Smith, N. J. Doran, W. Forysiak, and F. M. Knox, J. Lightwave Technol. 15, 1808 (1997).

7. D. J. Richardson, L. Dong, R. P. Chamberlin, A. D. Ellis, T. Widdowson, and W. A. Pender, Electron. Lett. 32, 4 (1996).

8. L. F. Mollenauer, Optical Ampliers and Their Applications, OSA Technical Digest Series (Optical Society of America, 2001), paper OMA2.

9. J. D. Ania-Castanon, Opt. Express 12, 4372 (2004).

10. J. D. Ania-Castanon, V. Karalekas, P. Harper, and S. K. Turitsyn, Phys. Rev. Lett. 101, 123903 (2008).

11. J. M. Dudley, L. P. Barry, P. G. Bollond, J. D. Harvey, and R. Leonhardt, Opt. Fiber Technol. 4, 237 (1998). 